\title{
Melanoma With Comedo-Like Openings: A Rare Dermoscopic Finding
}

\author{
Zorica Đorđević Brlek ${ }^{1}$, Jaka Radoš², Mirna Bradamante², Ružica Jurakić Tončić
}

1 Department of Dermatology and Venereology, Pula General Hospital, Pula, Croatia
2 University of Zagreb School of Medicine, Department of Dermatology and Venereology, University Hospital Centre Zagreb, Zagreb, Croatia

Key words: melanoma, dermoscopy, comedo-like openings

Citation: Đorđević Brlek Z, Radoš J, Bradamante M, Jurakić Tončić R. Melanoma with comedo-like openings: a rare dermoscopic finding. Dermatol Pract Concept. 2019;9(2):148-149. DOI: https://doi.org/10.5826/dpc.0902a12

Accepted: October 22, 2018; Published: April 30, 2019

Copyright: $\odot 2019$ Đorđević Brlek et al. This is an open-access article distributed under the terms of the Creative Commons Attribution License, which permits unrestricted use, distribution, and reproduction in any medium, provided the original author and source are credited.

Funding: None.

Competing interests: The authors have no conflicts of interest to disclose.

Authorship: All authors have contributed significantly to this publication.

Corresponding author: Ružica Jurakić Tončić, MD, University of Zagreb School of Medicine, Department of Dermatology and Venereology, University Hospital Centre Zagreb, Šalata 4, 10000 Zagreb, Croatia. Email: rjtoncic@gmail.com

\section{Introduction}

Seborrheic keratosis-like melanomas (SK-like melanomas) are difficult to diagnose and easily overlooked, especially among elderly patients with multiple clinically typical SKs which are usually not dermoscopically assessed and are removed with destructive measures without histopathological confirmation [1]. Therefore, systematic dermoscopic evaluation of all SKlike lesions should be made in order to avoid misdiagnoses, delayed treatment, and medicolegal consequences.

The term SK-like melanoma refers to melanoma that clinically and/or dermoscopically looks like SK, even if it is verrucous on histopathology report [1]. Comedo-like openings are predominantly found in SK, less frequently in Unna nevi, and very rarely in malignant melanoma, and they histopathologically correlate with keratin plugs within dilated follicular openings [1].

\section{Case Presentation}

We present a case of a 65-year-old woman with a lesion on her back that presented as ugly duckling sign. It was asymmetrical, nonulcerated, $5 \mathrm{~mm}$ in diameter, and slightly elevated with 3 different colors (dark brown, light brown, and gray; Figure 1). Dermoscopy showed striking asymmetry in shape, color, and structure, with gray-brown blotches, large light brown and pinkish structureless area, and unevenly distributed and sharply defined brown globules of varying size and shape grouped in asymmetrical clusters which resembled comedo-like openings (Figure 2). Complete excision was performed and histopathology revealed melanoma, Breslow thickness $1 \mathrm{~mm}$ with SK-like features and no signs of perineural or intravascular invasion (Figure 3).

\section{Conclusions}

SK-like melanoma can be dermoscopically very challenging and in case of any suspicion, histopathological confirmation is mandatory [1]. Although dermoscopic criteria have been described for differentiating melanocytic and nonmelanocytic skin lesions, some features can be confusing if simultaneously present in the same lesion [1]. Since comedo-like openings represent a typical feature of SK, they might mislead the clinician to a wrong diagnosis. In a study of melanomas 
resembling SK, Carrera et al [1] found the presence of comedo-like openings in $30.6 \%$ cases, as well as milia-like cysts in $22.4 \%$, yellowish keratin in $31.3 \%$, and scaly and hyperkeratotic surface in $33.6 \%$ [1]. Dermoscopy allows correct diagnosis of melanoma in more than $80 \%$ of clinically SK-like melanomas [1]. The main dermoscopic features identified as helpful are blue-black sign, blue-white veil, pseudopods or streaks, and pigment network [1]. Combining features of polarized light and nonpolarized light dermoscopy is beneficial since nonpolarized permits visualization of structures located in the upper skin layers, while polarized permits visualization of deeper structures which then enhances the conspicuity of different structures and may help reduce the rate of diagnostic errors [2].

In our case the diagnosis was straightforward due to melanomasuggestive dermoscopic clues. SK-like melanomas represent a potential diagnostic pitfall; therefore, dermatologists should be aware of "errors of perception," referring to a possibility of being drawn to one prominent but potentially misleading SK dermoscopic feature, while other subtle clues of melanoma in the visual field can be overlooked [2]. Complete dermoscopic evaluation of all quadrants of the lesion and careful analysis of all criteria should be undertaken to ensure that they are all consistent with the final diagnosis [2].

\section{References}

1. Carrera C, Segura S, Aguilera P, et al. Dermoscopic clues for diagnosing melanomas that resemble seborrheic keratosis. JAMA Dermatol. 2017;153(6):544-551.

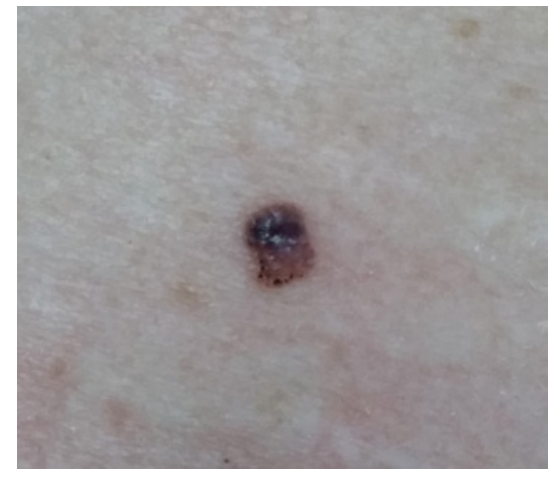

Figure 1. Clinical finding of malignant melanoma: asymmetrical, nonulcerated, $5 \mathrm{~mm}$ in diameter, and slightly elevated with 3 different colors (dark brown, light brown, and gray). [Copyright: @2019 Đorđević Brlek et al.]

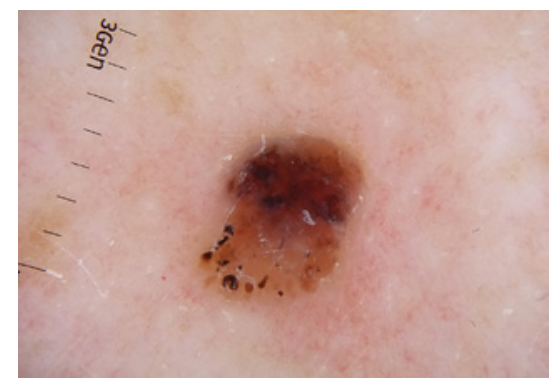

Figure 2. Dermoscopic finding of malignant melanoma: asymmetrical in shape, color, and structure, with gray-brown blotches, large light brown and pinkish structureless area, unevenly distributed and sharply defined brown globules of varying size and shape grouped in asymmetrical clusters that resemble comedo-like openings. [Copyright: C2019 Đorđević Brlek et al.]

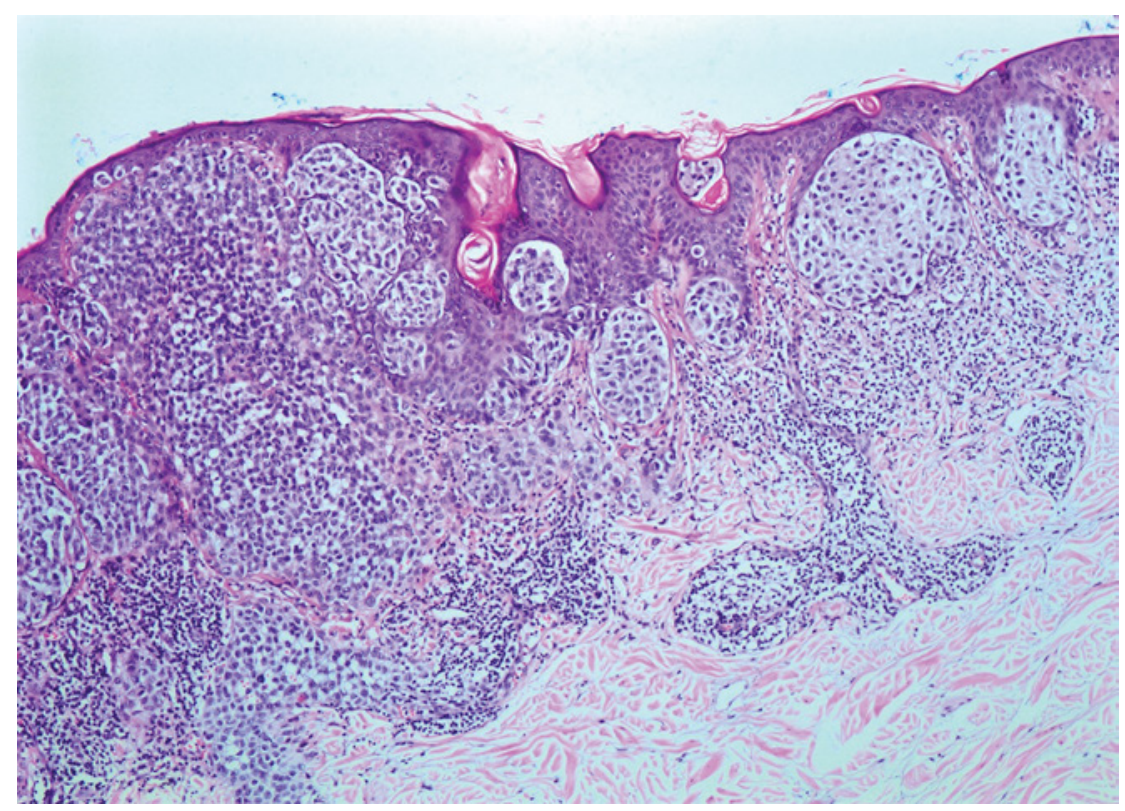

Figure 3. Histopathology of malignant melanoma: proliferation of atypical melanocytes singly and in nests within the epidermis, papillary and superficial portion of reticular dermis with smaller nests of atypical melanocytes located within follicular ostia filled with keratin plugs (hematoxylin and eosin, ×10). [Copyright: (C2019 Đorđević Brlek et al.]

2. Braga JC, Scope A, Klaz I, Mecca P, Spencer P, Marghoob AA. Melanoma mimicking seborrheic keratosis: An error of perception precluding correct dermoscopic diagnosis. J Am Acad Dermatol. 2008;58(5):875-880. 